\title{
A quantitative analysis of cerebrospinal fluid flow in post-traumatic syringomyelia
}

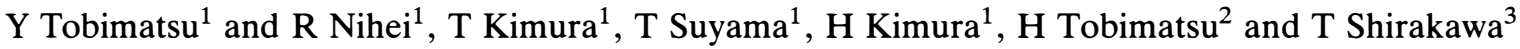 \\ ${ }^{1}$ Department of Orthopaedic Surgery, National Rehabilitation Center for the Disabled Hospital, PO Box 359, \\ 4-1 Namiki Tokorozawa City, Saitama Prefecture; ${ }^{2}$ Department of Rehabilitation, Fuchu Hospital, ${ }^{3}$ Siemens \\ Asahi Meditic, Japan
}

Cerebrospinal fluid (CSF) flow within the syrinx in post-traumatic syringomyelia was studied by cardiac-triggered phase images of magnetic resonance imaging (MRI) to investigate the relationship between CSF flow in the syrinx and patients' symptoms.

Keywords: post-traumatic syringomyelia; spinal cord injury; cerebrospinal fluid flow; MRI imaging

\section{Introduction}

Sixteen patients with post-traumatic syringomyelia were investigated. Nine had had a cervical spinal cord injury and seven a thoracic SCI. Thirteen had a complete spinal cord injury and three incomplete. Cardiac-triggered phase transverse images of the spinal cord including the syrinx were obtained by a modified sequence based on the gradient echo method, FLASH, using a $1.5 \mathrm{~T}$ superconductive MR imager. CSF flow in the subarachnoid space and in the syrinx were calculated from the phase modulation linear to CSF velocity.

Eleven of the patients including eight cervical spinal cord injured and three thoracic spinal cord injured had CSF flow in their syrinx. All the patients except one had symptoms such as severe pain (six cases), sensory disturbance (five cases), motor deterioration (five cases). In the five cases without CSF flow in the syrinx, four had no symptoms. The flow pattern was synchronised with the heart beats.

The CSF in the syrinx is correlated with the symptoms of syringomyelia. The investigation of the CSF flow in the syrinx may be valuable to determine the prognosis of the patients with post-traumatic syringomyelia. The cardiac-triggered phase method of MRI is simple and is useful to calculate CSF flow.

The purpose of this work was to investigate the relationship between the clinical features of posttraumatic syringomyelia and the CSF flow in the syrinx. In the past, post-traumatic syringomyelia was thought to be a rare complication of traumatic spinal cord injury. However, since MRI has come into popular use, it is known that post-traumatic syringomyelia is not rare. According to another of our studies, of patients with post-traumatic spinal cord injury admitted to the National Rehabilitation Center for the Disabled, 8.7\% had syringomyelia as shown by MRI. ${ }^{1}$

The CSF flow can be recognised on MR images by a void signal but this is not quantitative. Phase image may be used to calculate the flow rate in the subarachnoid space and the syrinx to investigate the relationships to the clinical features. ${ }^{2}$

\section{Materials}

We investigated 16 patients with post-traumatic syringomyelia. We defined the syrinxes that were clearly-bordered, with low intensity on T1 weighted imaging and high intensity on $\mathrm{T} 2$ weighted imaging, if longer than or equal to five segments as having syringomyelia.

Nine cervical cord injured patients and seven thoracic were included in the study. Fourteen were male and two were female. Thirteen who were completely paralysed (Frankel Grade A) and three who had incomplete paraplegia (Frankel Grade C) were included. The age at injury ranged from 12 to 45 years (average 22.1 years). The period between the injury and the MRI investigation ranged from 1 year 4 months to 27 year and 4 months (average 5 years and 6 months). The causes of trauma were traffic accident in 10 patients, sports accidents in four, and other causes in two patients.

\section{Methods}

We used a superconductive MRI machine made by Siemens, Magnetom 1.5 T. Modified sequence was used based on the gradient echo technique, FLASH (fast low angle shot). In order to evaluate slow flow perpendicular to the slice selected, we made the gradient amplitude of slice selection pulse larger, and in addition, the period of the gradient longer. By only making the gradient amplitude of slice selection pulse larger, phase modulation caused by the flow parallel to the slice becomes negligible. ${ }^{2}$ 
The phase shift, or $\mathrm{d} \phi$, caused by the following formula;

$$
\begin{aligned}
\mathrm{d} \phi & =\zeta_{0}^{\mathrm{t}} \mathrm{rvGtdt} \\
& =1 / 2 \mathrm{rvGt}^{2}
\end{aligned}
$$

where $d \phi=$ phase shift, $r=$ gyromagnetic ratio, $G=$ slice gradient, and $\mathrm{t}=$ the a additional time of the slice gradient (ms). The ' $r$ ', ' $v$ ', ' $t$ ' are the constants of this formula, so that the phase shift ' $\mathrm{d} \phi$ ' has a linear relationship to the velocity ' $v$ '. (Figure 1)

The imaging condition was that each patient was positioned supine on the surface coil, using the cardiac triggering mode, and 13 images of transverse spinal cord proximal to the injured level including syrinx were taken. Each slice was: thickness, $5 \mathrm{~mm}$; matrix size, 256 by 256 ; TE, $18 \mathrm{~ms}$; acquisitions, 1 to 2 times. TR was dependent on the length of cardiac cycle, because 13 cine images obtained during each cardiac cycle $(600-700 \mathrm{~ms})$ were equally spaced.

\section{Results}

Eleven patients including eight who had had a cervical spinal cord injury and three an injury of the thoracic cord, had a flow in their syrinxes (Table 1).

All except one patient had symptoms associated with syringomyelia: pain in six patients, sensory disturbance

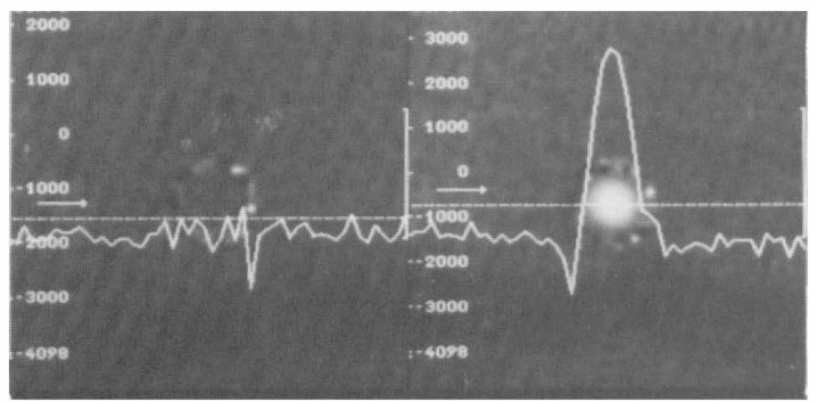

Figure 1 Phase image. Left: water in a tube at rest. Right: the water in the tube flows and the phase of the water in the tube modulates the phase of the surrounding water that does not flow in six patients, muscle weakness in five patients. The duration from the onset of their trauma to the time of the investigation averaged 6 years, and ranged from 9 months to 27 years and 4 months (Table 1 ).

The syrinxes spread to above $\mathrm{C} 2$ segment in nine out of the 11 patients. The longitudinal length of the syrinxes ranged from five segments spreading only to the proximal segments of the traumatically damaged segment to almost the whole of the cord from the obex to the conus medullaris. All spinal cords containing syrinxes were expanded (Figure 2).

The phase images and a diagram of the CSF velocity of the subdural and the intrasyrinx space at each delay time of the same patient are shown in Figures 3 and 4. The peak to the caudal flow occurred in the cardiac

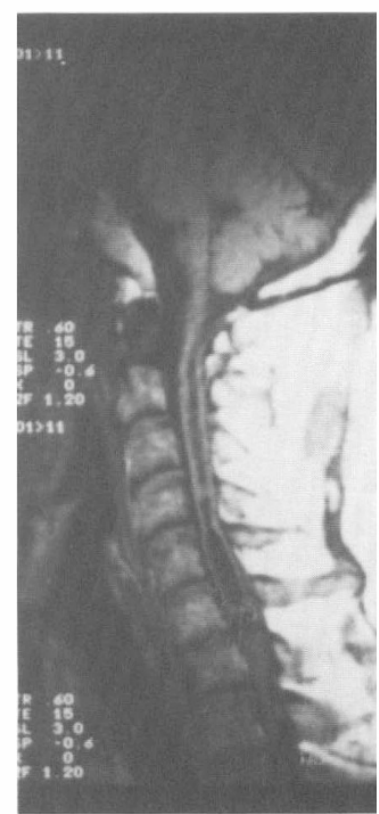

Figure $2 \mathrm{~T} 1$ weighted image of MR of the patient number (TR/TE; 600/15 ms). The syrinx spreads from the C7 segment, his traumatically damaged level, to the obex. The investigation was done 3 years 6 months after the accident. His symptoms were cervical and shoulder pain and hypaesthesia of the left side of the $\mathrm{C} 1$ to the $\mathrm{C} 7$ segment

Table 1 Patients with flow

\begin{tabular}{rrrrrrr}
\hline No. & SCI & Age & Period & Syrinx & Symptom & $\begin{array}{c}\text { Caudal peak flow } \\
\mathrm{cm} \mathrm{s}^{-1}\end{array}$ \\
\hline 1 & C5 & 16 & 1y 4 months & C2-C6 & Pain & 0.43 \\
2 & C6 & 17 & 5y 11 months & C2-C6 & Motor $\downarrow$ & 4.55 \\
3 & C6 & 22 & 4y 7 months & C2-T10 & Sensory $\downarrow$ & 1.77 \\
4 & C6 & 12 & 27y 4 months & C2-T10 & Sensory motor $\downarrow$ & 0.79 \\
5 & C7 & 37 & 3y 6 months & OB-C7 & Pain sensory $\downarrow$ & 2.06 \\
6 & C7 & 29 & 3y 9 months & OB-T10 & Pain sensory $\downarrow$ & 0.33 \\
7 & C7 & 16 & 5y 8 months & C2-T10 & Sensory motor $\downarrow$ & 0.86 \\
8 & C8 & 18 & 4y 2 months & C2-C7 & Pain & 0.44 \\
9 & T4 & 22 & 3y 5 months & C1-T10 & Pain & 0.78 \\
10 & T4 & 20 & 96-T10 months & C6-T12 & Motor $\downarrow$ & 0.67 \\
11 & T10 & 45 & 5y 8 months & T5-T12 & 0.28 \\
\hline
\end{tabular}




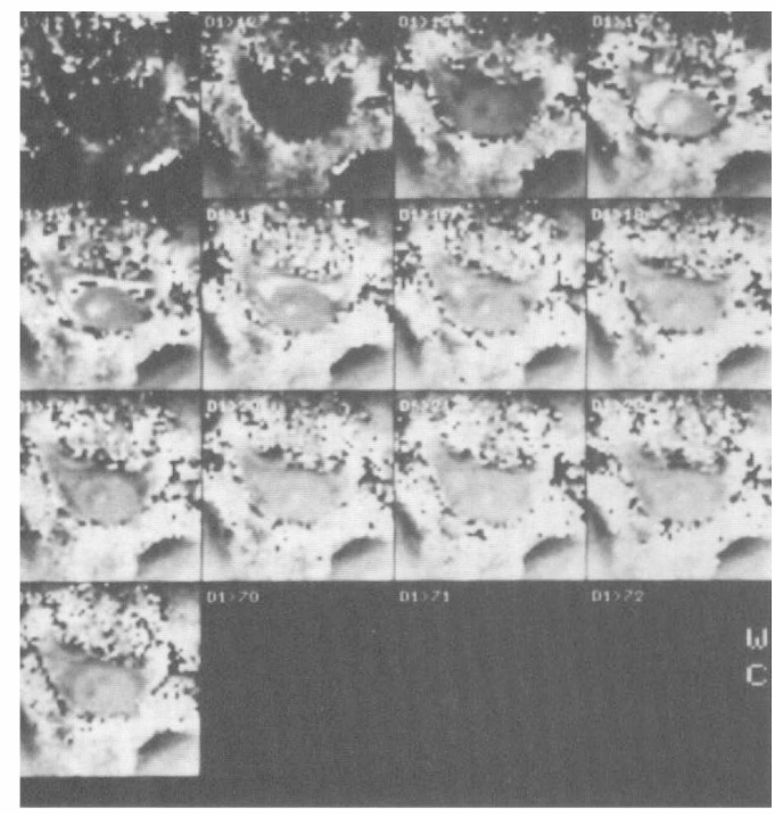

Figure 3 The phase image of CSF flow of the same patient as in Figure 2. It is arranged from left to right, from up to down. The upper part of each image is the anterior and the lower portion the posterior aspect. The intensity of spinal cord does not vary, but the intensity around the spinal cord and syrinx within the cord varies synchronously with the cardiac cycle. High intensity represents caudal flow and low, cranial flow

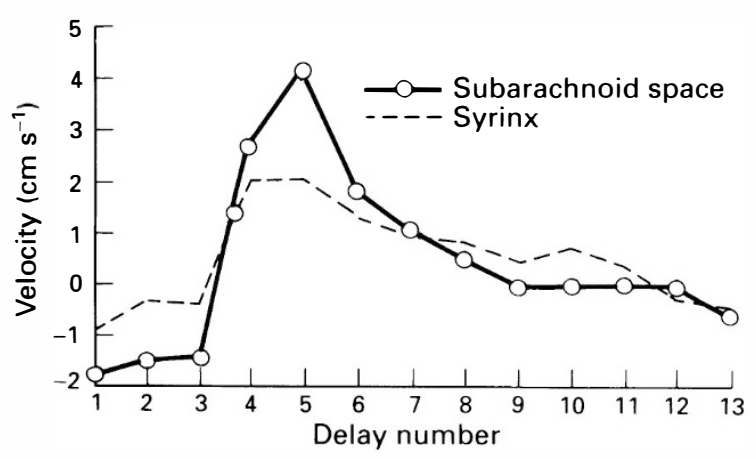

Figure 4 Diagram of the CSF velocity of the subdural and the intrasyrinx space of each delay time of the same patient as in Figures 2 and 3. The upward direction of the $Y$ axis is the caudal flow and the downward direction is the cranial flow. The $\mathrm{X}$ axis is the trigger delay number

systolic period, and moved in the cranial direction in the cardiac diastolic period. The cranial flow did not have a sharp peak. The patterns of flow were the same in the subarachnoid space and in the syrinx, but the velocity in the subarachnoid space was faster than it was in the syrinx. The flow pattern of other patients who had the flow in the syrinxes was almost the same, but some patients did not have a clear cranial flow and the flow in the subarachnoid space could not be observed, because of spinal cord swelling. The caudal peak velocity in the syrinxes ranged from $0.28 \mathrm{~cm} \mathrm{~s}^{-1}$ to $4.55 \mathrm{~cm} \mathrm{~s}^{-1}$, and was $1.28 \mathrm{~cm} \mathrm{~s}^{-1}$ on the average.

Five patients including one with a cervical cord injury and four with a thoracic 1 cord injury did not have a detectable flow in their syrinx (Table 2). Four of these patients had no symptoms associated with the syringomyelia. The symptoms in one patient who had no flow detected in his syrinx were backache, brachialgia, muscle weakness distal to T8 segment, and originally incomplete paralysis. The symptoms in this patient appeared just before the investigation, 7 years and 6 months after the accident and they progressed. The duration from the accident to the investigation ranged from 1 year 9 months to 8 years and 8 months, with a mean of 4 years and 7 months. Only one patient had a syrinx reaching cranially to $\mathrm{C} 2$ segment. The syrinxes in the other patients were distal to $\mathrm{C} 6$ segment. The length varied. Every spinal cord was swollen.

\section{Discussion}

The quantitative analysis of the CSF flow by MRI Before the development of MRI, research into CSF dynamics was conducted by myelography ${ }^{3,4}$ or by manometric techniques, ${ }^{5}$ by means of intradural punctures. Both methods were invasive and harmful and were not easy to perform. By either method, the axial CSF flow could be measured synchronously with the cardiac cycle. ${ }^{6,7}$ However, the CSF flow velocity could not be calculated, as the sensitivity was poor.

There are some methods to evaluate CSF flow by MRI. Muellar ${ }^{8}$ analyzed T2 change associated with flow, but the calculation was complicated and $\mathrm{T} 1$ and $\mathrm{T} 2$ of the substance which has velocity to calculate should be previously known. $\mathrm{Abe}^{9}$ used a saturation band method, which is an easy one and permits vision of the movement directly, but the sensitivity and accuracy were poor.

The phase method is based on phase modulations which are in proportion to the velocity of the spin under the conditions of the same gradient amplitude and duration. This method has several merits: the signal intensity and $\mathrm{T} 1$ and $\mathrm{T} 2$ values have no influence on the phase modulations; the flow direction can be ascertained; and the calculation of the velocity is simple.

Its demerits, however, are that motion artefacts greatly affect the phase images; normal imaging gradients will produce phase shifts, and phase modulation is caused not only by velocity, but also by different

Table 2 Patients without flow

\begin{tabular}{lccccc}
\hline No. & SCI & Age & Period & Syrinx & Symptom \\
\hline 1 & C6 & $17 \mathrm{y}$ & 8y 8 months & C1-C6 & - \\
2 & T4 & $16 \mathrm{y}$ & $2 \mathrm{y} 9$ months & T2-T8 & - \\
3 & T5 & $22 \mathrm{y}$ & $2 \mathrm{y} 2$ months & T2-T7 & - \\
4 & T5 & $17 \mathrm{y}$ & 7y 6 months & C6-T5 & Pain \\
& & & & Sensory $\downarrow$ & Motor $\downarrow$ \\
5 & T8 & $27 \mathrm{y}$ & 1y 9 months & C6-T10 & - \\
\hline
\end{tabular}


substances (such as fatty tissue) having different resonant frequencies, that is, a chemical shift.

These demerits can be easily overcome by using the phase of nearby static spin as a reference or by the subtraction method where flow encoding phase images are subtracted by non-encoding phase images which can remove the effect of the normal imaging gradients on the image; chemical shift effect can also be avoided by setting TE time, thus the spins of different chemical species are in-phase.

\section{Flow in the syrinx}

In our results of the measurement of flow in a syrinx, we found that the flow was to and fro synchronously with the cardiac cycle, and that the caudal flow velocity changed rapidly, and had a peak. In contrast, the cranial flow velocity changed slowly and had no peak. The pattern is the same as the CSF flow in the subarachnoid space. ${ }^{2}$ The to and fro pattern of CSF flow has been reported already by some authors. ${ }^{2,9,10,11}$ The brain expansion synchronous with the cardiac cycle propels the CSF from the cranium to the subarachnoid space in the spinal canal to generate a to and fro pattern of the CSF. ${ }^{12}$

In our study, the CSF in the syrinx was found to flow caudally during the systolic phase of the cardiac cycle, and cranial flow or no flow occurred during the diastolic phase. We feel that the syrinx or cyst, resulting from trauma, has a CSF flow that is synchronous with the cardiac cycle when the syrinx is connected to the central canal. The fact that patients who had a flow in their syrinxes had syrinxes spreading above C2 (nine patients out of 11) and patients who had no flow in the syrinx had the syrinx caudal to C6 (four patients out of five), and that there was no correlation with flow existence and the length of syrinxes assists a hypothesis of the connection between the syrinx and the central canal of the spinal cord.

\section{Flow in a syrinx and the symptoms associated with syringomyelia}

According to our results, we can say that the flow in a syrinx is related to the symptoms. From the point of view of the symptoms, almost all patients with a flow in their syrinx have symptoms, and almost all patients without a flow have no symptoms. The chief complaint of those with post-traumatic syringomyelia is severe pain, which becomes worse according to change of posture, sneezing, coughing or straining, all of which cause an increase in the pressure in the syrinx. We presume that these facts explain the relationship between the pressure of flow and the symptoms.

Whilst the CSF flow is clear in a caudal direction, flow in the cranial direction is not obvious and in some patients is small. Therefore we suspect that the caudal flow volume and the pressure in the syrinx is greater than the cranial flow's volume and the pressure from the syrinx. Further, the central canal is so narrow that the CSF entering the syrinx cannot flow back easily. Thus it would appear that a check valve mechanism between the syrinx and the central canal exists, so that when the volume and the pressure in the syrinx increase, pain and other symptoms appear. The chief complaint of patients who have a post-traumatic syrinx is pain. This symptom may be caused by a pressure increase in the syrinx. We suspect that the CSF flow in the syrinx is the important factor contributing to the symptom of pain.

\section{Flow in a syrinx and its expansion}

According to our results, a syrinx with a flow extended up to and beyond the C2 segment. We cannot determine the cause of such an extension. Foo et al ${ }^{13}$ have speculated that the syrinx is formed by the pooling of CSF in the degenerating space resulting from trauma. The patient whom they studied had come to autopsy and there was a small cyst at the site of trauma; it extended for only two spinal segments. Thus they were unable to pursue the progression of the symptoms and the growth of the syrinx. McLean et $_{\text {al }}{ }^{14}$ reported that the syrinx that forms at the site of degeneration or bleeding from trauma expands by raising the internal pressure caused by the obstruction of the subarachnoid space due to the trauma. Williams ${ }^{15}$ has suggested that the difference of pressure between the subarachnoid space and the syrinx causes the suck and slosh effect that expands the syrinx.

To know this, it is necessary to observe the development of a developing syrinx. Knowing when the flow in the syrinx appears, and comparing the flow pattern above the segment and caudally to the traumatically damaged segment are also necessary to clarify the cause of the development of post-traumatic syringomyelia.

\section{Conclusion}

Eleven patients out of 16 who had post-traumatic syringomyelia had the flow in their syrinxes investigated by MR phase imaging. Almost all of the patients with a flow in their syrinxes had symptoms associated with syringomyelia, and almost all of those without a flow had no symptom, with one exception. Almost all of the syrinxes with flow spread cranially as far as the C2 segment, and those without flow did not.

\section{References}

1 Tobimatsu $\mathrm{H}$ et al. Magnetic resonance imaging of spinal cord in chronic stage. Rinsho Seikei Geka 1991; 26: 1173-1182.

2 Tobimatsu Y et al. A quantitative analysis of cerebrospinal fluid flow in post-traumatic syringomyelia. J Jpn Orthop Assoc 1991; 65: 505-516.

3 Bouley G. Pulsatile movements in the CSF pathways. $\mathrm{Br} J$ Radiol 1969; 2: 193-216.

4 Barton L et al. Cerebrospinal fluid pulsations at myelography. A video-densitometric study. Radiology 1974; 110: 579-587.

5 Adolph RJ et al. Origin of cerebrospinal fluid pulsation. Am J Physiol. 1967; 212: 840-846.

6 du Boulay et al. Specialization broadens the view: the significance of a CSF pulse. Clin Radiol 1972; 23: 401-409.

7 Dardenne G et al. Cerebrospinal fluid pressure and pulsatility. Eur Neurol 1969; 2: 193-216.

8 Muellar E et al. Quantification of pulsatile flow in MRI by an 
analysis of T2 changes in ECG-Gated multiecho experiments. Magn Reson Med 1986; 3: 331-335.

9 Abe $\mathrm{H}$ et al. Magenetic resonance cineflow imaging of cerebrospinal flow in syringomyelia. Neuroradiology 1991; 33 (suppl): 173-175.

10 Njemantze PC, Beck OJ. MR-gated intracranial CSF pulsatile flow. Am J Neuroradiol 1989; 10: 77-80.

11 Mascalchi $\mathrm{M}$ et al. Cardiac-gated phase MR imaging of aquaductal CSF flow. J Comput Assist Tomogr 1988; 12: 923-926.
12 Feinberg DA, Mark AS. Human brain motion and cerebrospinal fluid circulation demonstrated with MR velocity imaging. Radiology 1987; 163: 793-799.

13 Foo $\mathrm{D}$ et al. A case of post-traumatic syringomyelia neurological findings after 1 year of cystic drainage. Paraplegia 1989; 27: 63-69.

14 McLean DA et al. Post-traumatic syringomyelia. J Neurosurg 1973; 39: 485-492.

15 Williams B. On the pathogenesis of syringomyelia: a review. J R Soc Med 1980; 73: 798-806. 